\title{
Bounded Generalized Random Linear Operators
}

\author{
Nguyen Thinh* \\ Department of Mathematics, VNU University of Science, 334 Nguyen Trai, Hanoi, Vietnam \\ Received 03 May 2017 \\ Revised 30 June 2017; Accepted 15 September 2017
}

\begin{abstract}
In this paper we are concerned with bounded generalized random linear operators. It is shown that each bounded generalized random linear operator can be seen as a set-valued random variable. The properties of some special bounded generalized random linear operators and the random resolvent set of generalized random linear operators are investigated.

Keywords: Random linear operator, random bounded linear operator, generalized random linear mapping, bounded generalized random linear operator, set-value random variable, random resolvent set, random regular value.
\end{abstract}

AMS Subject Classification 2000: Primary 60H25; Secondary 60H05, 60B11, 45R05.

\section{Introduction}

Let $X, Y$ be separable Banach spaces and $(\Omega, F, P)$ be a probability space. By a random mapping (or a random operator) from $\mathrm{X}$ to $\mathrm{Y}$ we mean a rule that assigns to each element $x \in X$ a $\mathrm{Y}$-valued random variable (r.v.). Mathematically, a random mapping defined from $\mathrm{X}$ to $\mathrm{Y}$ is simply a mapping $A: X \rightarrow L_{0}(\Omega, Y)$ where $L_{0}(\Omega, Y)$ stands for the space of all Y-valued random variables (r.v.'s). If $S=[a, b]$ is a interval of the real line then $F=\{F(t)\}_{t \in[a, b]}$ is said to be a Y-valued stochastic process.

The interest in random mappings has been arouse not only for its own right as a random generalization of deterministic mappings as well as a natural generalization of stochastic processes but also for their widespread applications in other areas. Research in theory of random mappings has been carried out in many directions including random linear mappings which provide a framework of stochastic integral, infinite random matrix (see e.g. [2, 5, 11], [14-19]), random fixed points of random operators, semi groups of random operators and random operator equations (e.g. [3], [6], [10]-[16] and references therein).

\footnotetext{
* Tel.: 84-983181880

Email: thinhj@gmail.com

https//doi.org/ 10.25073/2588-1124/vnumap.4191
} 
Under the original definition, a random mapping $\mathrm{F}: S \rightarrow L_{0}(\Omega, Y)$ is a rule that transforms each deterministic input $x \in S$ into a random output Fx. Taking into account that inputs may be also random, a generalized random mapping is defined as a mapping $\Phi: S \rightarrow L_{0}(\Omega, Y)$, where $\mathrm{S}$ is a subset of $L_{0}(\Omega, X)$.

A random mapping $\mathrm{X} \rightarrow L_{0}(\Omega, Y)$ which is linear and bounded is called a random linear bounded operator (see $[11,14,15,17])$ and a generalized random mapping $L_{0}(\Omega, X) \rightarrow L_{0}(\Omega, Y)$ which is strongly linear and bounded is called a bounded generalized random linear operator. In Section 2 the one-one corresponding between random linear bounded operators and bounded generalized random linear operators is discussed. It is shown that every random linear bounded operator from $X$ to $Y$ admits a unique extension which is a bounded generalized random linear operator from $L_{0}(\Omega, X) \rightarrow L_{0}(\Omega, Y)$. Reversely, if $\Phi: L_{0}(\Omega, X) \rightarrow L_{0}(\Omega, Y)$ is a bounded generalized random linear operator then the mapping $\$$ restricted on $X$ will be a random linear bounded operator.

By [17], the random mapping A : $\mathrm{X} \rightarrow L_{0}(\Omega, Y)$ is a random linear bounded operator if and only if there exists almost surely uniquely a mapping $\mathrm{T}$ from $\Omega$ to set of all linear bounded operators from $\mathrm{X}$ to $\mathrm{Y}$ such that for each $\mathrm{x} \in \mathrm{X}$ we have $A x(\omega)=T(\omega) x$ a.s. Thus a random linear bounded operator from $X \in Y$ can be regarded as a family $T$ indexed by $\omega \in \Omega$ satisfying for each $\mathrm{x} \in \mathrm{X}$ the mapping $\omega \mapsto T(\omega) x$ is measurable.

Section 3 is concerned with a different form of random linear bounded operators and bounded generalized random linear operators. Theorem 3.1, 3.2 show that a random linear bounded operator (or a bounded generalized random linear operator) from $\mathrm{X}$ to $\mathrm{Y}$ can be regarded as a measurable setvalued mapping from $\mathrm{Q}$ to set of all linear bounded operators from $\mathrm{X}$ to $Y$.

As an application, the properties of some special bounded generalized random linear operators and random resolvent set of generalized random linear operators are investigated (Theorem 3.3, 3.4, 3.6).

\section{Random bounded operators and bounded generalized random linear operators}

In this section, some definitions and typical results on random bounded operator, bounded generalized random linear operator are listed and discussed. For more details, we refer the reader to $[14,17,18,19]$.

Throughout this paper, $(\Omega, F, P)$ is a complete probability space, $\mathrm{X}, \mathrm{Y}$ are separable Banach spaces. A measurable mapping $\xi$ from $(\Omega, F)$ into $(X, B(X))$ is called a $X$-valued random variable. The set of all $\mathrm{X}$-valued random variables is denoted by $L_{0}(\Omega, X)$. We do not distinguish two $\mathrm{X}$ random variables which are equal almost surely.

$L_{0}(\Omega, X)$ is a metric space under the metric of convergence in probability. If a sequence $\left(u_{n}\right)$ in $L_{0}(\Omega, X)$ converges to $\mathrm{u}$ in probability then we write $\mathrm{p}-\lim \xi_{n}=\xi$ 
Definition 2.1. ([14, 17])

- By a random mapping A from $\mathrm{X}$ to $\mathrm{Y}$ we mean a mapping from $\mathrm{X}$ into $L_{0}^{Y}(\Omega)$

- By a random linear mapping $\mathrm{A}$ from $\mathrm{X}$ to $\mathrm{Y}$ we mean a mapping from $\mathrm{X}$ into $L_{0}^{Y}(\Omega)$ satisfying for every $\lambda_{1}, \lambda_{2} \in \mathbb{R}, x_{1}, x_{2} \in X$ we have

$$
A\left(\lambda_{1} x_{1}+\lambda_{2} x_{2}\right)=\lambda_{1} A\left(x_{1}\right)+\lambda_{2} A\left(x_{2}\right) \text { a.s. }
$$

- A random linear mapping $A: X \rightarrow L_{0}^{Y}(\Omega)$ is said to be a random opeartor if it is continuous and is said to be bounded (or random bounded operator) if there exists a real-valued random variable $k(\omega)$ such that for each $\mathrm{x} \in \mathrm{X}$

$$
\|A x(\omega)\| \leq k(\omega)\|x\| \quad \text { a.s. }
$$

Noting that the exceptional set in (1) may depend on $x$.A random bounded operator is a random operator but in general, a random operator needs not be bounded. For examples of random operators, random bounded operators and unbounded random bounded operators, we refer to $[14,17,19]$. It is easy to prove the following Theorem which is a little bit more general than a result in [17].

Theorem 2.2. A random mapping A : $X \rightarrow L_{0}^{Y}(\Omega)$ is a random bounded operator if and only if there is an almost surely uniquely mapping $\mathrm{T}: X \rightarrow L_{0}^{Y}(\Omega)$ such that for each $x E X$,

$$
A x(\omega)=T(\omega) x \text { a.s. }
$$

For the sake of convenience, we denote the a.s. uniquely determined mapping $T(w)$ in the Theorem above by $[A](w)$. So, for each $\mathrm{x} \in \mathrm{X}$, we have

$$
A x(\omega)=[A](\omega) x \text { a.s. }
$$

Definition 2.3. $\quad 1$. Let $\mathcal{M}$ be a subset of $L_{0}(\Omega, X)$. By a generalized random mapping $\Phi$ defined on $\mathcal{M}$ with values in $\mathrm{Y}$ we mean a mapping $\Phi: \mathcal{M} \rightarrow L_{0}(\Omega, X)$. As usual the domain $\mathcal{M}$ of $\Phi$ is denoted by $D \Phi$.

2. A subset $\mathcal{M} \subset L_{0}(\Omega, X)$ is said to be a random linear subspace if for every $u_{1}, u_{2} \in M, \xi_{1}, \xi_{2} \in L_{0}(\Omega)$ we have $\xi_{1} u_{1}+\xi_{2} u_{2} \in \mathcal{M}$.

3. Let $\mathcal{M} \subset L_{0}(\Omega, X)$ be a random linear subspace. By a generalized random linear operator (g.r.l.o) defined on $\mathcal{M}$ with values in Y we mean a strongly linear mapping $\Phi: \mathcal{M} \rightarrow L_{0}(\Omega, Y)$ i.e. if $u_{1}, u_{2} \in \mathcal{M}, \xi_{1}, \xi_{2} \in L_{0}(\Omega)$ then

$$
\Phi\left(\xi_{1} u_{2}+\xi_{2} u_{2}\right)=\xi_{1} \Phi u_{1}+\xi_{2} \Phi u_{2}
$$

4. A generalized random linear operator $\Phi: L_{0}(\Omega, X) \rightarrow L_{0}(\Omega, Y)$ is said to be bounded if there exist a random variable k s.t. $\|\Phi u\| \leq k\|u\|$ a.s. $\forall u \in L_{0}(\Omega, X)$. 
It should be noted that the notion of g.r.l.o. has been introduced in [18] where X, Y are Hilbert spaces.

If $\Phi: L_{0}(\Omega, X) \rightarrow L_{0}(\Omega, Y)$ is a bounded generalized random linear operator then the restricted operator $\left.\Phi\right|_{X}: X \rightarrow L_{0}(\Omega, Y)$ is a random bounded linear operator. Reversely, if $A: X \rightarrow L_{0}(\Omega, Y)$ is a random bounded linear operator then by [17], A admits uniquely an extention $\Phi: L_{0}(\Omega, X) \rightarrow L_{0}(\Omega, Y)$ which is a bounded generalized random linear operator and moreover for each $u \in L_{0}(\Omega, X)$

$$
\Phi(u)(\omega)=[A](\omega) u(\omega) \text { a.s. }
$$

Combining this with Theorem 2.2, it is easy to have the following Theorem.

Theorem 2.4. A generalized random mapping $\Phi: L_{0}(\Omega, X) \rightarrow L_{0}(\Omega, Y)$ is a bounded g.r.l.o. if and only if there is an almost surely uniquely mapping $\mathrm{T}: \Omega \rightarrow L(X, Y)$ such that for each $u \in L_{0}^{X}(\Omega)$

$$
\Phi u(\omega)=T(\omega) u(\omega) \text { a.s. }
$$

For the sake of convenience, we denote the a.s. uniquely determined mapping $\mathrm{T}(\omega)$ as in the Theorem above by $[\Phi](\omega)$. So, for each $u \in L_{0}^{X}(\Omega)$, we have

$$
\Phi u(\omega)=\Phi(\omega) u(\omega) \text { a.s. }
$$

The set-valued analysis is used as main technique in proofs in the next chapter. Next we list some notions and typical results relating to set-valued r.v. to be used later on.

Let $(\mathrm{E}, \mathrm{d})$ be a separable metric space. Denote $2^{E}$ the collection of all subsets of $\mathrm{E}, B(E)$ the set of all Borel measurable sets in (E, d). A mapping $F: \Omega \rightarrow 2^{\mathrm{E}}$ is called a set-valued function. A r.v. $\mathrm{f}$ : $\Omega \rightarrow \mathrm{E}$ is said to be a measurable selections of $\mathrm{F}$ if $f(\omega) \in F(\omega), \forall \omega \in \Omega$

Definition 2.5. ([7], Definition 1.1) Let $\mathrm{F}: \Omega \rightarrow 2^{\mathrm{E}} \backslash \emptyset$ be a set-valued function.

(a) $\mathrm{F}$ is said to be strongly measurable if for every $\mathrm{C} \subseteq \mathrm{E}$ closed, we have $\mathrm{F}^{-1}(\mathrm{C})=\{\omega \in S: F$ $(\omega) \cap C=\emptyset\} \in F$

(b) $\mathrm{F}$ is said to be measurable or set-valed random variable if for every $\mathrm{C} \subseteq \mathrm{E}$ open, we have $\mathrm{F}$ $-1(\mathrm{C})=\{\omega \in \mathrm{T}: \mathrm{F}(\omega) \cap \mathrm{C}=\emptyset\} \in F$

(c) If $\mathrm{F}$ is measurable then it is called a set-valued random variable.

(d) $\mathrm{F}$ is said to be graph measurable if $\operatorname{Gr}(\mathrm{F})=\{[\omega, x] \in \mathrm{T} \times \mathrm{E}$ :

$\mathrm{x} \in \mathrm{F}(\omega)\} \in \mathrm{F} \times \beta(\mathrm{E})$.

Theorem 2.6. [7] (Theorem 1.35, Proposition 2.3) Let $\mathrm{F}: \Omega \rightarrow{ }_{2}^{\mathrm{X}} \backslash \emptyset$ s.t. $F(w)$ is closed set for every $\omega \in \Omega$. Then the following statements are all equivalent. 
1. For every $\mathrm{C} \in B(\mathrm{E}), \mathrm{F}^{-1}(\mathrm{C}) \in \Omega$;

2. $F$ is strongly measurable;

3. $F$ is measurable;

4. For every $x \in \mathrm{E}$, the mapping $\omega \rightarrow d(x, F(\omega))$ is measurable;

5. $F$ is graph measurable.

6. There exists a sequence $\left\{f_{n}\right\}_{n \geq 1}$ of measurable selections of F, s.t. for every $w \in \Omega, F(w)=\overline{\left\{f_{n}(w)\right\}_{n \geq 1}}$. Such a sequence $\left(f_{n}\right)$ is called dense measurable selections of $\mathrm{F}$.

Given a set-valued function $F: \Omega \rightarrow 2^{\mathrm{E}} \backslash\{\varnothing\}$, we denote $\mathrm{SF}=\{\mathrm{f} \in$

$\mathrm{L}_{0}(\Omega, \mathrm{E}): \mathrm{f}(\omega) \in \mathrm{F}(\omega)$ a.s. $\}$.

Theorem 2.7. ([7], implied from Theorem 3.9) Let $F, G: \Omega \rightarrow 2 E \backslash \varnothing$ are closed set-valued r.v.'s. If $S_{F}=S_{G}$ then $F(\omega)=G(\omega)$ a.s.

\section{Main results}

Let $\Phi: L_{0}^{X}(\Omega) \rightarrow L_{0}^{Y}(\Omega)$ be a bounded g.r.l.o. It is known that in general the mapping

$[\Phi]: \Omega \rightarrow \mathrm{L}(\mathrm{X}, \mathrm{Y})$

$\omega \rightarrow[\Phi](\omega)$

is not Borel-measurable. However, the following Theorem shows that this mapping is measurable in term of set-valued measurable.

Let $\mathrm{A}: \mathrm{D}(\mathrm{A}) \subseteq \mathrm{X} \rightarrow \mathrm{Y}$ be an arbitrary mapping. Denote $\operatorname{Gr}(\mathrm{A})=\{[\mathrm{x}, \mathrm{y}] \in \mathrm{X} \times \mathrm{Y}: \mathrm{x} \in$ $D(\mathrm{~A})\} \subseteq 2^{\mathrm{X} \times \mathrm{Y}}$. Note that $\mathrm{X} \times \mathrm{Y}$ is a separable Banach space under the norm

$$
\|(x, y)\|_{X \times Y}=\|x\|_{X}+\|y\|_{Y}, \forall x \in X, y \in Y
$$

Theorem 3.1. Let $\Phi: L_{0}^{X}(\Omega) \rightarrow$ set of all mapping from $\Phi$ to $Y$ (needs not be measurable). Then $\Phi$ is a bounded g.r.l.o. if and only if there is an almost surely uniquely mapping $T: \Omega \rightarrow L(X$, Y) such that for each $u \in L_{0}^{X}(\Omega)$,

$$
\Phi u(\omega)=T(\omega) u(\omega) \quad \text { a.s. }
$$

and the mapping

$$
\begin{aligned}
& \operatorname{Gr}(T): \Omega \rightarrow 2^{X \times Y} \\
& \omega \mapsto G r(T(\omega))
\end{aligned}
$$

is a closed set-valued r.v.

Because of the corresponding between random bounded operator and bounded g.r.l.o., the Theorem above is equivalent to the Theorem below.

Theorem 3.2. A : $X \rightarrow$ set of all mapping from $\Omega$ to $\mathrm{Y}$ (needs not be measurable). Then $\mathrm{A}$ is a random bounded operator if and only if there is an almost surely uniquely mapping $T: \Omega \rightarrow L(X, Y)$ such that for each $x \in X$, 
$A x(\omega)=T(\omega) x \quad$ a.s.

and the mapping

$$
\begin{aligned}
& \operatorname{Gr}(T): \Omega \rightarrow 2^{X \times Y} \\
& \omega \mapsto \operatorname{Gr}(T(\omega))
\end{aligned}
$$

is a closed set-valued r.v.

Proof. Sufficiency condition: Let A be a random bounded operator and $\left(\mathrm{x}_{\mathrm{n}}\right)$ be a condense sequence of $\mathrm{X}$. Put $\mathrm{T}=[\mathrm{A}]$. We construct a sequence of mapping $\left(\omega_{n}\right)$ from $\Omega$ to $X \times Y$ as follows:

$$
\omega_{n}(w)=\left(x_{n}, T(\omega) x_{n}\right)
$$

$A x_{n}$ is measurable so $\omega_{n}$ is also measurable. Now we will check that for each $\omega \in \Omega$ the sequence $\left(\omega_{n}(u)\right)_{n}$ is a dense set in $\operatorname{Gr}(T(\omega))$. Indeed, let $\omega$ be an arbitrary element of $\operatorname{Gr}(T(\omega))$, then there exists $x \in X$ such that $\omega=(x, T(\omega) x)$. Since $\left(x_{n}\right)$ is dense in $X$ then there exists a subsequence $x_{n^{\prime}}$ converging to $x$. The boundedness of $T(u)$ implies that the sequence $\left(T(\omega) x_{n^{\prime}}\right)$ converges to $T(\omega) x$ and thus $\omega_{n^{\prime}}=\left(x_{n^{\prime}}, T(\omega) x_{n^{\prime}}\right)$ converges to $\omega=(x, T(\omega) x)$. So $\left(\omega_{n}\right)$ are dense measurable selections of $\operatorname{Gr}(T)$. By Theorem 2.6, the closed set-valued mapping $\operatorname{Gr}(T)$ is measurable.

Necessity condition: assume $\mathrm{T}: \Omega \rightarrow L(X, Y)$ is mapping such that (6) holds and the closed setvalued mapping $\operatorname{Gr}(T)$ is measurable. By Theorem 2.2, it remains to prove $A x$ is measurable. By Theorem 2.6, there exists a measurable sequence $\left(\omega_{n}\right)$ such that for every $\omega \in \Omega, \omega_{n}(\omega)=\left(u_{n}\right.$ $\left.(\omega), T(\omega) u_{n}(\omega)\right)$ and $\left(\omega_{n}(\omega)\right)$ is dense in $\operatorname{Gr}(T(\omega))$. The measurability of $\omega_{n}$ leads to the measurability of $u_{n}$ and $A u_{n}=T(\cdot) u_{n}(\cdot)$. Let $x \in X$ and fix $\omega \in \Omega$. There exist a subsequence $\left(\omega_{n^{\prime}}(\omega)\right)$ of $\left(\omega_{n}(\omega)\right)$ that converges to $\omega(\omega)=(x, T(\omega) x)$. If $\omega_{n^{\prime}}(\omega)=\left(u_{n^{\prime}}(\omega), T(\omega) u_{n^{\prime}}\right)$ then $\left(u_{n^{\prime}}(\omega)\right)$ converges to $x$. This implies the sequence $u_{n}(\omega)$ is dense in $X$ for every $\mathrm{u} \omega \in \Omega$. Now let $x \in X$ and fix $e>0$, we will construct a random variables $v$ such that $\|v(u)-x\| \leq e$ for every $w$ and $\mathrm{T}(\cdot) v(\cdot)$ is also measurable. Indeed, for each $n \in \mathbb{N}$, we define a set by induction: $B_{n}=\left\{\omega:\left\|u_{n}(\omega)-x\right\| \leq e\right\}$ and $C_{n}=B_{n} \backslash \bigcup_{k=1}^{n-1} B_{k}$. It is not difficult to verify that $C_{n}$ are disjoint measurable sets and $C_{n} \subseteq \Omega$. Let $v(\omega)=\sum_{n=1}^{\infty} 1_{C_{n}} u n(\omega)$, it is easy to see that $\|v(u)-x\| \leq e$ for every $\omega \in \Omega$ and $T(\cdot) v_{n}(\cdot)$ is measurable. From this we can construct a sequence of measurable random variables $v_{n}$ such that $\left\|v_{n}(\omega)-x\right\| \leq 1 / n$ for every $\omega \in \Omega$ and $T(\cdot) v_{n}(\cdot)$ is measurable. Combining with the boundedness of $T(\omega)$ we can conclude that $T(\cdot) x$ is measurable. The theorem is proved completely.

Theorem 3.3. Let $\Phi: L_{0}^{X}(\Omega) \rightarrow L_{0}^{Y}(\Omega)$ be a bounded g.r.l.o. If $\Phi$ is an injective mapping then for almost surely $\omega \in \Omega$, the mapping $[\Phi](\omega) \in L(X, Y)$ is injective.

Proof. For each $\omega \in \Omega$, let $\quad N(\omega)=\{x \in X:[\Phi](\omega) x=0\} \subset X . \quad$ Put $X 0=\{(x, 0): x \in X\} \subset X \times Y$. Observe that, for each $\omega \in \Omega, N(\omega) \equiv G r([\Phi(\omega)]) \cap X 0$. It is 
obvious that the closed set-valued mapping $\omega \mapsto X 0$ is a graph measurable. Thus the closed setvalued mapping $\omega \mapsto N(\omega)$ is graph measurable. By Theorem 2.6, there exists a sequence

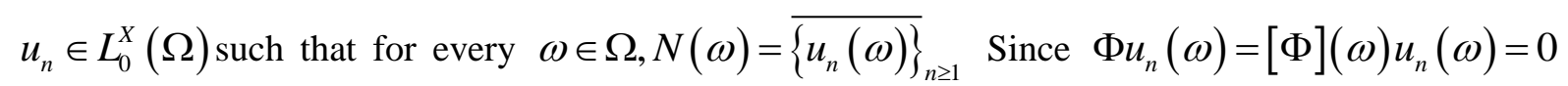
a.s. and $\Phi$ is injective, $u_{n}=0$ a.s. So there exists a measurable set $\Omega_{1}$ s.t. $P\left(\Omega_{1}\right)=1$ and $u_{n}(\omega)=0$ for every $\omega \in \Omega_{1}$ Hence $N(\omega)=\{0\}$ for every $\omega \in \Omega_{1}$. In other words, for almost surely $\omega \in \Omega$, the mapping $[\Phi](\omega): X \rightarrow Y$ is injective.

Denote $\mathcal{L}_{c}(X, Y)$ the set of all closed linear operators $T: D(T) \subseteq X \rightarrow Y$

Theorem 3.4. Let $\Phi^{-1}: \mathcal{D}(\Phi) \subset L_{0}^{X}(\Omega) \rightarrow L_{0}^{Y}(\Omega)$ be a g.r.l.o. If $\Phi$ is injective, surjective and $\Phi^{-1}: L_{0}^{Y}(\Omega) \rightarrow L_{0}^{X}(\Omega)$ is a bounded g.r.l.o. then there exists an almost surely uniquely mapping $T: \Omega \rightarrow \mathcal{L}_{c}(X, Y)$ such that

1. For almost surely u $E$ Q, the mapping $T(\omega): \mathcal{D}(T(\omega)) \subset X \rightarrow Y$ is injective, surjective and $T(\omega)^{-1} \in L(Y, X)$.

2. The mapping

$$
\begin{aligned}
& \operatorname{Gr}(T): \Omega \rightarrow 2^{X \times Y} \\
& \omega \mapsto G r(T(\omega))
\end{aligned}
$$

is a closed set-valued r.v.

3.

$$
\begin{aligned}
& \mathcal{D}(\Phi)=\left\{u \in L_{0}(\Omega, X): u(\omega) \in \mathcal{D}(T(\omega)) a . s .\right\}, \\
& \Phi u(\omega)=T(\omega)(u(\omega)) a . s . \forall u \in \mathcal{D}(\Phi), \\
& G r(\Phi)=\left\{v \in L_{0}^{X \times Y}(\Omega): v(\omega) \in G r(T(\omega))\right\}
\end{aligned}
$$

Proof. 1. The mapping $\Phi^{-1}: \mathcal{D}(\Phi) \subset L_{0}^{Y}(\Omega) \rightarrow L_{0}^{X}(\Omega)$ is a bounded g.r.l.o. Since $\Phi^{-1}$ is injective, by Theorem 3.3, the mapping $\left[\Phi^{-1}\right](\omega) \in L(Y, X)$ is injective for almos $\omega \in \Omega$. For each $\omega \in \Omega$, put $T(\omega)=\left(\left[\Phi^{-1}\right](\omega)\right)^{-1}$ then $T(\omega) \in \mathcal{L}_{c}(X, Y)$ since $\left[\Phi^{-1}\right](\omega) \in L(Y, X)$. It is obviuos that $T(\omega): \mathcal{D}(T(\omega)) \subset X \rightarrow Y$ is injective, surjective and and $T(\omega)^{-1}=\left(\left[\Phi^{-1}\right](\omega)\right)^{-1} \in L(Y, X)$

2. By Theorem 3.1, the closed set-valued mapping

$$
\operatorname{Gr}\left(\Phi^{-1}\right): \Omega \rightarrow 2^{Y \times X}
$$




$$
\omega \mapsto G r\left(\left[\Phi^{-1}\right](\omega)\right)
$$

is measurable. Thus it is easy to see that the closed set-valued mapping

$$
\begin{aligned}
& \operatorname{Gr}\left(\Phi^{-1}\right): \Omega \rightarrow 2^{Y \times X} \\
& \omega \mapsto G r(T(\omega))=\operatorname{Gr}\left(\left[\Phi^{-1}\right](\omega)\right)^{\text {transposed }}
\end{aligned}
$$

is also measurable.

3. It is easy to verify (11), (12) and (13).

The a.s. uniqueness of $\mathrm{T}$ is implied from (13) and Theorem 2.7.

\section{Definition 3.5.}

1. Let $\Phi: \mathcal{D}(\Phi) \subset L_{0}^{X}(\Omega) \rightarrow L_{0}^{X}(\Omega)$ be a g.r.l.o.

$\lambda \in L_{0}^{\mathbb{C}}(\Omega)$ is said to be a random regular value of $\$$ if the mapping $\lambda I D-\Phi$ is injective, surjective and the mapping $(\lambda I D-\Phi)^{-1}: L_{0}^{X}(\Omega) \rightarrow L_{0}^{X}(\Omega)$ is a bounded g.r.l.o. Where $I D$ is the identity mapping on $L_{0}^{X}(\Omega)$

2. The set of all random regular values of $\Phi$ is called random resolvent set of $\Phi$ and is denoted by $\rho(\Phi)$

Theorem 3.6. Let $\Phi: \mathcal{D}(\Phi) \subset L_{0}^{X}(\Omega) \rightarrow L_{0}^{X}(\Omega)$ be a g.r.l.o. If the random resolvent set of $\Phi$ is not empty then there is an almost surely uniquely mapping $U: \Omega \rightarrow \mathcal{L}_{c}(X, X)$ such that

1. The mapping

$$
\begin{aligned}
& \operatorname{Gr}(U): \Omega \rightarrow 2^{X \times Y} \\
& \omega \mapsto G r(T(\omega))
\end{aligned}
$$

Is a closed set-valued r.v

2.

$$
\begin{aligned}
& \mathcal{D}(\Psi)=\left\{u \in L_{0}(\Omega, X): u(\omega) \in T(U(\omega)) a . s .\right\}, \\
& \Psi u(\omega)=T(\omega)(u(\omega)) \text { a.s. } \forall u \in \mathcal{D}(\Phi), \\
& G r(\Phi)=\left\{v \in L_{0}^{X \times Y}(\Omega): v(\omega) \in G r(T(\omega))\right\}
\end{aligned}
$$

and we have

$$
\rho(\Phi)=\left\{\lambda \in L_{0}^{\mathbb{C}}(\Omega): \lambda(\omega) \in \rho(T(\omega)), \forall \omega \in \Omega\right\}
$$


Proof. Assume $\lambda \in \rho(\Phi)$. Put $\Psi=\lambda I D-\Phi$. Then $\Psi$ is injective, surjective and $\Psi^{-1}=L_{0}^{X}(\Omega) \rightarrow L_{0}^{X}(\Omega)$ is a bounded g.r.l.o. By Theorem 3.4, there exists an almost surely uniquely mapping $U: \Omega \rightarrow \mathcal{L}_{c}(X, Y)$ such that

1. For almost surely $w \in \Omega$, the mapping $U(\omega): \mathcal{D}(U(\omega)) \subset X \rightarrow Y$ is injective, surjective and $U(\omega)^{-1} \in L(X, X)$.

2. The mapping

$$
\begin{aligned}
& \operatorname{Gr}(U): \Omega \rightarrow 2^{X \times Y} \\
& \omega \mapsto G r(U(\omega))
\end{aligned}
$$

is a closed set-valued r.v.

3.

$$
\begin{aligned}
& \mathcal{D}(\Psi)=\left\{u \in L_{0}(\Omega, X): u(\omega) \in \mathcal{D}(U(\omega)) a . s .\right\}, \\
& \Psi u(\omega)=U(\omega)(u(\omega)) \text { a.s. } \forall u \in \mathcal{D}(\Psi), \\
& G r(\Psi)=\left\{v \in L_{0}^{X \times Y}(\Omega): v(\omega) \in G r(U(\omega))\right\}
\end{aligned}
$$

Now for each w G Q, put $\mathrm{T}(\omega)=\mathrm{A}(u) i d-U(\omega)$, where $i d$ is the identity mapping on $X$. It is not difficult to verify (10), (11), (12), (13), (14).

\section{Acknowledgments}

This work was supported by Vietnam National University under Grant QG.14.12

\section{References}

[1] R. Carmona and J.Lacroix, Spectral theory of random Schrdinger operators, Birkhuser, Boston, MA, 1990.

[2] A.A. Dorogovtsev, On application of gaussian random operator to random elements, Theor. veroyat. i priment, 30:812-814, 1986.

[3] A.A. Dorogovtsev, Semigroups of finite-dimensional random projections, Lith. Math. J., 51(3):330-341, 2011.

[4] N. Dunford and J.T. Schwarts, Linear operators Part II, Interscience Publishers, NewYork, 1963.

[5] H.W. Engl, M.Z. Nashed, and M. Zuhair, Generalized inverses of random linear operators in banach spaces, J. Math. Anal. Appl., 83:582-610, 1981.

[6] H.W. Engl and W. Romisch, Approximate solutions of nonlinear random operator equations: Convergence in distribution, Pacific J. Math., 120:55-77, 1985.

[7] S. Hu and N.S. Papageorgiou, Handbook of multivalued analysis. Vol. I, Mathematics and Its Applications, Kluwer Academic Publishers, Dordrecht, 1997.

[8] M. Ledoux and M. Talagrand, Probability in Banach spaces, Springer-Verlag, 1991. 
[9] N. Hussain N. Shahzad, Deterministic and random coincidence point results for f-nonexpansive maps, J.Math.Anal. Appl., 323:1038-1046, 2006.

[10] M.Z. Nashed and H.W. Engl, Random generalized inverses and approximate solutions of random equations, in Bharucha-Reid A.T. (Ed.), Approximate Solution of random equations, Elsevier, North-Holland, New YorkAmsterdam, 1979, pp. 149-210.

[11] A.V. Skorokhod, Random Linear Operators, Reidel Publishing Company, Dordrecht, 1984.

[12] V.S. Sunder, Functional Analysis: Spectral Theory, Birkhauser Verlag, Boston-Berlin, 1998.

[13] G. Taraldsen, Spectral theory of random operators: The energy spectrum of the quantum electron in a disordered solid, Ph.D. dissertation, Norwegian University of Science and Technology, Trondheim, Norway, 1992.

[14] D.H. Thang, Random operators in banach spaces, Probab. Math. Statist., 8:155-157, 1987.

[15] D.H. Thang, Random mappings on infinite dimensional spaces, Stochastics An International Journal of Probability and Stochastic Processes: formerly Stochastics and Stochastics Reports, 64:51-73, 1998

[16] D.H. Thang and P.T. Anh, Random fixed points of completely random operators, Random Oper. Stoch. Equ., 21:1-20, 2013.

[17] D.H. Thang and N. Thinh, Random bounded operators and their extension, Kyushu J.Math., 57:257-276, 2004.

[18] D.H. Thang and N. Thinh, Generalized random linear operators on a hilbert space, Stochastics An International Journal of Probability and Stochastic Processes: formerly Stochastics and Stochastics Reports, 84(6):1040-1059, 2013.

[19] D.H. Thang, N. Thinh, and T.X. Quy, Generalized random spectral measures, Journal of Theoretical Probability, 27:676-600, 2014. 